\title{
Prólogo - Avances y retrocesos de la soberanía alimentaria
}

\author{
Alberto Acosta
}

\section{SciELO Books / SciELO Livros / SciELO Libros}

ACOSTA, A. Prólogo - Avances y retrocesos de la soberanía alimentaria In: ESTEBAN, D., ARTACKER, T., and LIZANO, R., coords. Cambio climático, biodiversidad y sistemas agroalimentarios: avances y retos a 10 años de la Ley Orgánica del Régimen de la Soberanía Alimentaria en Ecuador [online]. Quito: Editorial Abya-Yala, 2020, pp. 7-15. ISBN: 978-9978-10-466-8. http://doi.org/10.7476/9789978105689.0001.

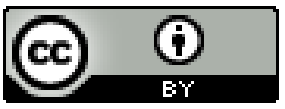

All the contents of this work, except where otherwise noted, is licensed under a Creative Commons Attribution 4.0 International license.

Todo o conteúdo deste trabalho, exceto quando houver ressalva, é publicado sob a licença Creative Commons Atribição 4.0.

Todo el contenido de esta obra, excepto donde se indique lo contrario, está bajo licencia de la licencia Creative Commons Reconocimento 4.0. 


\section{Prólogo \\ Avances y retrocesos de la soberanía alimentaria}

Alberto Acosta ${ }^{1}$

La Constitución ecuatoriana de 2008, aprobada masi-

vamente en comicios generales, es un documento que rompe moldes y señala posibles caminos a seguir para las naciones en una época en la que el planeta nos empieza a pasar factura por la destrucción de sus ciclos vitales. Por ello ha sido estudiada con lupa por expertos de distintos lugares del mundo. (...) Una de las novedades más significativas es la introducción de los conceptos de la soberanía alimentaria y del ally kawsay, el buen vivir

Javier Carrera (Cofundador de la Red de Guardianes de las Semillas).

En la Asamblea Constituyente de Montecristi, uno de los temas de recurrente debate fue el de las soberanías. Sí, en plural. En este ámbito, la soberanía implica no solo conservar el territorio dentro de los cánones del Estado-nación de siglos pasados. Hablar de soberanías en la actualidad es soñar en territorios que gocen del mayor grado posible de autonomía económica, política, energética, cultural y sobre todo alimentaria. Territorios en donde prime el respeto y

1 Economista ecuatoriano. Profesor universitario. Exministro de Energía y Minas. Expresidente de la Asamblea Constituyente (2007-2008). 
defensa de los derechos de las personas, de las comunidades, de los pueblos y de los Estados, tanto como los Derechos de la Naturaleza. Inclusive cabe comenzar a pensar colectivamente en la construcción de una soberanía latinoamericana en el marco de la Patria Grande.

Visto el tema de las soberanías con este prisma amplio, la descentralización y la autonomía forman parte de un proceso de emancipación y recuperación de la soberanía. No se trata de una mera arquitectura institucional en el ámbito territorial. Este esfuerzo autonómico, por lo demás, no pretende fragmentar el país. Se busca potenciar la soberanía desde abajo, desde lo local — barrios, comunidades, municipios, prefecturas - afectando tanto la concentración de los recursos fiscales en pocas regiones, así como la concentración de la riqueza en pocas manos, como parte de un proceso de reencuentro con la Naturaleza. Se trata, en suma, de que los y las habitantes de un país o región, tanto como las comunidades, controlen cada vez más directamente y de manera autónoma el diseño de sus sociedades y el manejo de sus vidas. Esta visión de la soberanía en plural afecta privilegios, estructuras patriarcales, prácticas depredadoras y lógicas autoritarias.

De hecho, la soberanía alimentaria resulta un proyecto incómodo para los actores que impulsan la agroindustria y la agroexportación, es decir para la acumulación del capital, sea en el marco de cadenas de producción nacionales o transnacionales. También plantea la superación de opciones estatizantes o burocratizantes que limitan el papel del verdadero actor y gestor de la soberanía alimentaria en el ámbito de la producción de alimentos: el campesinado. Por lo tanto, resulta inaceptable esta visión para quienes piensan que pueden ayudar a superar las condiciones de pobreza de los agricultores con políticas asistencialistas diseñadas desde arriba, con el concurso muchas veces de tecnocracias incapaces de cambiar sus estrechos márgenes de comprensión y por ende de acción.

Con la soberanía alimentaria se cuestiona, entonces, el relato dominante que viene a la cabeza cuando se abordan los temas agrarios, 
cargados de un sinnúmero de estereotipos que muchas veces son falsos o al menos incompletos. Relatos únicos que subordinan la agricultura al capital imposibilitando la cristalización de alternativas, que bien sabemos existen en el mundo. De hecho, cuando hablamos de soberanía alimentaria ponemos en entredicho aquel discurso tecnocrático, que tanto fomentan los diversos gobernantes, que ven el tema agrario con los lentes de la productividad y la competitividad, casi siempre en clave del mercado internacional. A contrapelo de esas lecturas y prácticas la soberanía alimentaria se nutre de las visiones comunitarias que afloran en su relación de equilibro con la Pachamama.

De este tipo de reflexiones surgieron propuestas concretas en varios ámbitos en Montecristi. Estos reclamos para construir y reconstruir la soberanía se plasmaron en la Constitución del 2008, aprobada mayoritariamente por el pueblo ecuatoriano en las urnas el 28 de septiembre de dicho año. Allí, por ejemplo, en el artículo 281, se dispone que el Estado garantice la soberanía alimentaria, tema abordado también en los artículos 13, 262, 281, 282, 282, 304, $318,334,410,413,423 \ldots$. Esta propuesta constitucional emergió con fuerza desde el mundo indígena y campesino ecuatoriano, estrechamente vinculado a las visiones y planes de una de las organizaciones más grandes del planeta, como lo es la Vía Campesina; propuesta que supera en mucho lo que se conoce como seguridad alimentaria, que podría ser conseguida por el simple uso de la fuerza como se plantea y se ejecuta desde varias potencias hegemónicas.

La soberanía alimentaria — que no puede quedar en un simple saludo a la bandera-demanda la aplicación de una política integral que implica recuperar el control sobre la propia agricultura y la alimentación. Esto nos remite al derecho de los agricultores y las agriculturas, en especial de las pequeñas unidades campesinas, en ejercer el control sobre la agricultura, y al derecho de los consumidores y las consumidoras a ejercitar el control sobre la alimentación.

Diversificar, defender y fomentar la producción agrícola y pecuaria sostenible, proteger y aprovechar la biodiversidad de ecosistemas 
y microclimas, asoman como primeras tareas en esa dirección. Fomentar las capacidades productivas y de gestión de las comunidades campesinas e indígenas, los encadenamientos productivos y comerciales de unidades agrícolas y cooperativas campesinas existentes y la constitución de nuevas unidades de producción aglomeradas en función de este objetivo, constituye otro ámbito de acción fundamental. El objetivo es una agricultura más soberana, más incluyente, más proveedora de empleos y respetuosa del medio ambiente, que asegure la alimentación adecuada de toda la población, sin priorizar el mercado externo sacrificando estas metas vitales para la existencia de una sociedad.

Esto implica una estrategia adecuada de redistribución de la tierra; de protección, redistribución y distribución de los recursos hídricos (que por Constitución no pueden ser privatizados: artículos 282 y 318 ; en este último artículo se plantea inclusive la priorización para el uso del agua primero para los seres humanos y la soberanía alimentaria; agua que debe ser asumida en tanto elemento estratégico y patrimonial y en ningún caso como mercancia). Lo anterior presupone también el reconocimiento del territorio de los pueblos y las nacionalidades indígenas: territorio que no puede ser confundido como un simple factor de producción. Todo lo anterior se conseguirá estimulando el rescate de la tecnología tradicional y el desarrollo de las innovaciones y adaptaciones tecnológicas propias en el sector. La mayoría de los agricultores y campesinos, valorizando en primer lugar sus conocimientos y prácticas ancestrales, deben adquirir nuevos conocimientos, habilidades, actitudes y hasta valores que les permitan atender las crecientes demandas alimenticias.

Para lograr la soberanía alimentaria resulta urgente fortalecer la agroecología y la economía campesina, en base, entre otras medidas, a la eliminación del uso indiscriminado y creciente de agroquímicos, fomentando y estimulando la producción orgánica. Es indispensable diversificar la producción rural y realizar un decidido esfuerzo para enfrentar las causas estructurales de la pobreza rural con el fin de construir un mundo rural productivo plural e integra- 
do, que inclusive apunte a ruralizar las ciudades en tanto allí se recuperan niveles de sensatez ecológica y de cálida convivencia humana.

Otra acción fuerte de la soberanía alimentaria radica en una política de comercialización agropecuaria, que rompa con la red oligopólica de los intermediarios. Es obligatorio asegurar el abastecimiento y la distribución de alimentos, a través también de la regulación de sus precios, impulsando la comercialización directa en las ciudades, creando redes solidarias de abastecimiento y mercados locales.

Entre los muchos temas que componen esta estrategia de soberanía alimentaría, habrá que transformar el sistema educativo para superar el desencuentro entre lo que las escuelas rurales enseñan y aquello que realmente se necesita aprender, provocando en las escuelas de las urbes nuevas formas de entender la realidad del campo y cómo aproximarse a ella con respeto. Adicionalmente, son necesarias escuelas superiores de agricultura orientadas a atender las necesidades concretas de los productores rurales que requieren, en carácter prioritario, de tecnologías conviviales, es decir sencillas y de bajo costo, para que sean compatibles con los recursos que los campesinos disponen. Los servicios públicos de extensión rural tendrán que ajustarse a las demandas del campo y no al revés.

Promoviendo la soberanía alimentaria, hay que consolidar la defensa de los recursos genéticos, y la prohibición de la importación y uso de transgénicos. Esta regulación se plasmó en el artículo 401 de la Constitución, que:

Declara al Ecuador libre de cultivos y semillas transgénicas. (...) El Estado regulará bajo estrictas normas de bioseguridad, el uso y el desarrollo de la biotecnología moderna y sus productos, así como su experimentación, uso y comercialización. Se prohíbe la aplicación de biotecnologías riesgosas o experimentales.

Dicho esto, es indispensable ejercer plena soberanía sobre los recursos de la biodiversidad, consolidar la protección de los ecosistemas y establecer un sistema riguroso de respeto al conocimiento tradicional. 
La construcción de soberanía en el ámbito de los conocimientos en el agro, recuperando los saberes ancestrales, es prioritaria. En la sección sobre Biodiversidad de la Constitución, artículos 400 a 404, se construyó el marco referencial que debe inspirar el manejo del enorme patrimonio de uno de los países más mega biodiversos del planeta. La soberanía sobre los conocimientos ancestrales, dentro de la sección Ciencia, tecnología, innovación y saberes ancestrales, es otro reto constitucional, como reza el artículo 385.

Un punto clave es asegurar la libertad de las semillas que no pueden estar normadas de tal manera que se siga consolidando el dominio de pocos oligopolios, al tiempo que se subordina más y más la vida campesina a la lógica de acumulación del capital. No habrá soberanía alimentaria, ni soberanía sobre los recursos naturales, en el marco del respeto a los Derechos de la Naturaleza, mientras la tierra esté controlada por grupos y actividades que prioricen los negocios antes que la vida misma; es decir, no habrá soberanía mientras la tierra sea asumida como una simple mercancía.

Todo lo expuesto anteriormente sintetiza los elementos de lo que deberían ser parte para una gran transformación agraria. Tarea que no solo que está inconclusa, sino que, por el contrario, deberá empezar desde menos cero si se comprende que las políticas agrarias desplegadas desde la aprobación de la Constitución en el 2008 han estado orientadas a resolver los problemas de los agronegocios y a ampliar sus campos de acción, en una clara acción contraria a las demandas y necesidades del campesinado. Es inocultable que los gobiernos a los que nos referimos han priorizado en extremos los extractivismos en un esfuerzo por modernizar el capitalismo, demostrando -en la práctica y más allá de los discursos- que tanto progresismo como neoliberalismo caminan, quizás a momentos por andariveles formalmente diferentes, en la misma dirección: servir a la acumulación del capital.

El saldo es inocultable: el latifundio no ha sido discutido, la tierra no se ha "desconcentrado", las mejores áreas cultivables sir- 
ven al monocultivo de exportación y el agua de riego sigue captada prevalentemente por esas propiedades. Con las políticas del progresismo y más aún con el neoliberalismo, la tierra y el territorio no se convirtieron realmente en un derecho que abarque a diversas dimensiones de la vida, sino que quedaron reducidos a meros medios de explotación, importantes solo según el nivel de producción. Así la visión mercantilista sigue intacta, en donde grandes extensiones de tierra para agroexportación —incluyendo los agrocombustiblesdevienen en modelos de productividad, que bien sabemos no resuelven los temas de fondo, como lo es la alimentación adecuada y digna de toda la población. En cambio, la agricultura a pequeña escala es vista (por ignorancia de quienes así lo aseveran) como causal del empobrecimiento y del atraso del campo, y hasta es asumida como traba al desarrollo agrícola del país.

No nos olvidemos que el presidente Rafael Correa no aceptó la reforma agraria. No hizo cumplir los mandatos de la Constitución de 2008 que prohíben el acaparamiento de la tierra, así como el acaparamiento y la privatización del agua. Él llegó incluso a afirmar — desde su visión miope de competitividad y productividad desarrollistaque "la pequeña propiedad rural va en contra de la eficiencia productiva y de la reducción de la pobreza [...] repartir una propiedad grande en muchas pequeñas es repartir pobreza" (1 de octubre de 2011). Este desconocimiento de la materia explica su compromiso con los grandes propietarios. Con su cuestionamiento a la economía campesina inclusive se cerró - al menos por lo pronto — la puerta a ampliar y potenciar las existentes alternativas con las que se puede y debe renovar e innovar la agricultura en estrecho diálogo con los saberes ancestrales. Como no podía ser de otra forma, semejante accionar marginó a la soberanía alimentaria. No se garantizó el acceso a la tierra ni tampoco al agua, a las semillas, a las maquinarias, a los créditos, a las tecnologías, a los conocimientos... Además, se ignoró el potencial de los saberes ancestrales y comunitarios, puestos en grave riesgo con la firma del TLC con la Unión Europea; convenio que también acciona en la dirección contraria en otros campos de la soberanía alimentaria.... 
Partiendo de estas breves reflexiones cabe preguntarse no solo cuánto se ha avanzado desde Montecristi, que como anotamos no es mucho, por no decir definitivamente nada, sino cuáles son aquellos elementos clave para entender el proceso transcurrido desde hace más de una década de la expedición de la Ley Orgánica del Régimen de la Soberanía Alimentaria.

Ese es el objetivo del presente libro.

Las respuestas son contundentes. No solo que resulta insuficiente lo avanzado, sino que, en muchos casos, se ha caminado abiertamente en contra del mandato constitucional. Bastaría con tener presente el veto presidencial a dicha ley aprobada en la Asamblea Nacional, que de por si representaba un muy tímido intento de cumplir con dicho mandato, como se anota en el primer artículo de este libro.

En los siguientes artículos se aborda esta cuestión desde varios ángulos. La lectura de lo que ha sido la realidad de soberanía alimentaria es motivo de recurrente preocupación, anotando la necesidad de suspender la narrativa del triunfo normativo que contribuye a la desmovilización de los grupos que alentaron y alientan la soberanía alimentaria, en un esfuerzo que además nos permita comprender también las perversas derivas de la institucionalización de la participación en el proceso. Tampoco se dio paso a la soberanía alimentaria conjugando el verbo "sustentabilidad" en forma efectiva. Por el contrario, no se liberó a la sustentabilidad de tantas deformaciones que han conducido a su vaciamiento o si se quiere a su transformación en un concepto de elástico que sirve inclusive para calificar cuestiones que por definición no son ni pueden ser sustentables, como la minería, para citar apenas uno de los casos de uso más aberrantes.

Esas iniciales aproximaciones al tema se enriquecen y amplían con aportes que abordan las cuestiones fundamentales de alimentación, nutrición y comercialización, destacando el papel de las redes alternativas de alimentos, los circuitos alternativos de comercialización. Un asunto que se complementa discutiendo los problemas para 
el acceso a mercados de la agricultura familiar campesina, incorporando el ejercicio de los derechos culturales y colectivos.

En este libro, escrito por personas conocedoras de la materia, se incorporan preguntas vitales que tienen que ver con el papel de la soberanía alimentaria en la búsqueda de los Objetivos de Desarrollo Sostenible — de ninguna validez práctica, cabría anotar al margen- en tanto potencial aporte para enfrentar el colapso climático, reconociendo los riesgos que existen por la omnipresencia de los plaguicidas altamente peligrosos. Con algunas experiencias territoriales se cierra este aporte sustancial para seguir insistiendo en la construcción de una estrategia de soberanía alimentaria que rebase los simples discursos y que cristalice los avances constitucionales de Montecristi. Una tarea por demás pendiente.

Cerremos estas breves líneas resaltando la imperiosa necesidad de comprender el profundo contenido de esta visión de mundo desde abajo. Y para hacerlo nada mejor que repetir, como lo hace Gustavo Duch, uno de los grandes promotores de la soberanía alimentaria, aquel pensamiento africano que sintetiza lo que realmente debe movilizarse para hacer realidad la soberanía alimentaria: "mucha gente pequeña, en muchos lugares pequeños, cultivarán pequeños huertos...que alimentarán al mundo". 